\title{
THE INVERSE PROBLEM OF DETERMINING THE FILTRATION FUNCTION AND PERMEABILITY REDUCTION IN FLOW OF WATER WITH PARTICLES IN POROUS MEDIA
}

\author{
A.C. ALVAREZ, G. HIME, D. MARCHESIN, AND P. G. BEDRIKOVETSKY
}

\begin{abstract}
Deep bed filtration of particle suspensions in porous media occurs during water injection into oil reservoirs, drilling fluid invasion of reservoir production zones, fines migration in oil fields, industrial filtering, bacteria, viruses or contaminants transport in groundwater, etc. The basic features of the process are particle capture by the porous medium and consequent permeability reduction.

Models for deep bed filtration contain two quantities that represent rock and fluid properties: the filtration function, which is the fraction of particles captured per unit particle path length, and formation damage function, which is the ratio between reduced and initial permeabilities. These quantities cannot be measured directly in the laboratory or in the field; therefore, they must be calculated indirectly by solving inverse problems. The practical petroleum and environmental engineering purpose is to predict injectivity loss and particle penetration depth around wells. Reliable prediction requires precise knowledge of these two coefficients.

In this work we determine these quantities from pressure drop and effluent concentration histories measured in one-dimensional laboratory experiments. The recovery method consists of optimizing deviation functionals in appropriate subdomains; if necessary, a Tikhonov regularization term is added to the functional. The filtration function is recovered by optimizing a non-linear functional with box constraints; this functional involves the effluent concentration history. The permeability reduction is recovered likewise, taking into account the filtration function already found, and the functional involves the pressure drop history. In both cases, the functionals are derived from least square formulations of the deviation between experimental data and quantities predicted by the model.
\end{abstract}

Deep bed filtration, Suspension transport, Porous media, Inverse problem, Tikhonov regularization, Formation damage, System of convection-reaction equations

\section{INTRODUCTION}

Severe injectivity decline during sea- or produced water injection is a serious problem in offshore waterflood projects. This decline results from permeability impairment because the rock captures particles from the injected water. Reliable modeling-based prediction of injectivity decline is important for the design of injected water treatment or management by water filtering, injection of sea- and produced water combinations, etc.

During flow of water with suspended particles through porous media, the particles are gradually retained, reducing the permeability of the medium. This phenomenon is called deep bed filtration with formation damage. Formation damage can be induced by the penetration of drilling fluid into a reservoir. Other petroleum related applications for which filtration and formation damage are important include sand production control, fines migration, disposal of produced water in aquifers and deep bed filtration in gravel packs. Particle suspension filtration also occurs in industrial water filtering, in propagation through aquifers of contaminants (including viruses, bacteria, etc), and in other environmental processes. 
Mathematical models for suspension transport in porous media consist of an advectiondiffusion equation of particle mass balance with a particle capture term and Darcy's law accounting for permeability reduction due to particle retention (see [12], [19], [26], [32], [33], [38] and [44]). The models contain two empirical functions describing properties of the aqueous suspension and of the porous medium: the filtration function, i.e., the probability for a particle to be captured per unit particle path length, and the permeability reduction, i.e., the ratio between the reduced and the initial permeabilities. Particle deposition alters pore space geometry and hydraulic resistivity; in turn, pore geometry alters conditions for further deposition, so it is natural to take the deposited concentration as the basic independent variable for the filtration and formation damage functions.

Laboratory coreflood tests with particle suspension are carried out routinely in order to estimate injectivity decline during water injection into oil reservoirs (see [32] and [47]). In these one-dimensional experiments, the retained profile is non-uniform: in the laboratory, it is impossible to create a suspended-retained particle system with uniform deposited concentration. Therefore, the filtration and formation damage functions cannot be measured directly. However, it is possible to measure the time series of suspended particle effluent concentration and of pressure drop, and these two functions can be recovered indirectly from these experimental measurements by solving inverse problems for deep bed filtration and formation damage.

For modeling purposes, the filtration and damage functions are given suitable parametrizations that are compatible with the physical properties of the phenomenon. The filtration function reduces to a constant coefficient for the case of diluted suspensions; it can be calculated from the mean effluent concentration. The formation damage function is usually represented by a hyperbolic formula containing one empirical parameter - the so-called formation damage coefficient - which can be calculated from the pressure drop along the core ([19], [32], [47]). Alternatively, both coefficients can be calculated from pressure data at three core points ([5], [6]).

In this work, we present a more flexible method for determining the filtration and formation damage with any arbitrary form using parameter optimization. Differently from other methods, these quantities are not assumed to be constant, rather they are functions of the deposited particle concentration. We introduce regularization to generalize the procedure given in [18]. The recovery procedure for the filtration and permeability reduction functions presented in this work consists of optimizing certain functionals using the projection gradient method with box constraints developed in [8]. The functionals to be minimized are obtained from a least squares formulation taking into account the difference between experimental data and quantities predicted by the model. The box constraints reflect physical properties of the solution such as positivity and monotonicity. The functions obtained from the coreflood data may then be used for predicting well injectivity decline during water injection by solving the direct problem for deep bed filtration.

This paper is organized as follows. In Section 2, we present the dispersion-free deep bed filtration model with formation damage as a system of two hyperbolic equations for suspended and retained concentrations and a pressure balance equation. We also explain how to solve this system by integrating two families of ordinary differential equations. In Section 3 , we describe the optimization procedures we use to solve the two inverse problems. In the first one, we use the calculated effluent concentration history to define the functional that is minimized to determine the filtration function. In the second one, we use the deposition 
calculated using this recovered filtration function to predict the pressure drop history and we define the functional that is minimized to determine the permeability reduction function. In Section 4, we validate the recovery methods and we briefly examine the sensitivity of these inverse problems by means of synthetic data. In Section 5, we apply the method to experimental data and discuss the recovered functions as obtained by solving the two inverse problems.

\section{Flow OF WATER With PARTICLES IN POROUS MEDiA.}

In this section we present the physical model for the flow of water with suspended particles suffering retention in porous media. This model was developed in [5] based on [19]. Neglecting particle diffusion and dispersion, the mass balance equation for linear flow accounting for suspended and retained particles is

$$
\frac{\partial}{\partial t}(\phi c+\sigma)+U \frac{\partial c}{\partial x}=0
$$

Here the volumetric concentrations of suspended and deposited particles are respectively $c(x, t) \in[0,1]$ and $\sigma(x, t) \in[0, \phi]$. The dimensionless quantity $\phi \in[0,1]$ is called the rock porosity: it is the fraction of the rock volume available to the fluid. It is further assumed that the overall porous space is available for small particles, that size exclusion is not important, and that one particle plugs one pore.

We consider the flow of diluted suspensions, where the "particle-pore reaction" can be assumed to be of order one for small suspended concentrations, i.e., the retention rate is proportional to the suspended concentration $c$ :

$$
\frac{\partial \sigma}{\partial t}=\lambda(\sigma) U c .
$$

The dependence of the retention rate on $\sigma$ is expressed by $\lambda(\sigma)$, which is called the filtration function: it is the probability for a particle to be captured per unit length of the trajectory ([19], [37]). The retention rate $\partial \sigma / \partial t$ is proportional to the flow velocity $U$ ([19], [21]).

The filtration function is assumed to be independent of the retained particle concentration, i.e., constant in $\sigma$, for most laboratory tests on bacteria transport, where a diluted colloid flows during a short time corresponding to just a few injected pore volumes ([12], [29]). In this case, the retained concentration is low, and particle deposition does not alter the rock surface. In the case of longer flow periods or higher injected concentrations, we assume a Langmuir retention kinetics where the retention rate is proportional to the number of "vacancies", so that $\lambda$ depends linearly on $\sigma$ (the so-called blocking function, see [23] and [26]). In contrast, the random sequential adsorption (RSA) approach employs a filtration function that is a non-linear function of $\sigma$ (also in [23]).

During seawater flooding of oil reservoirs, a large amount of water is injected, corresponding to millions of times the volume of the damaged zone around the well, and the retained particles may occupy up to a $5-30 \%$ fraction of the porous volume near the well. Therefore, the structure of the pore space and surface where particles are retained changes completely during injection ([32], [35], [45], [47]), and the function $\lambda(\sigma)$ can become non-linear and even non-monotonic.

Remark 2.1. The retention rate in equation (2.2) can be interpreted in different ways. Several authors drop the velocity factor ([10], [29]), and $\lambda$ becomes the probability for particles to be captured per time unit. In this case, $\lambda$ is a function of velocity expressed in terms of 
dimensionless parameters. Nevertheless, in several important cases the form of this function suggests that $\lambda$ is proportional to $U$ ([28], [34]), and the retention kinetics equation becomes (2.2). In [19] and [2], it is assumed that the capture rate by "vacancies" is proportional to particle flux. In the dispersion-free case the flux is equal to $U c$, which also leads to (2.2).

We assume that the permeability reduction expressed by $k(\sigma)$ is due to particle retention, and that it is a decreasing function of the retained concentration ([32], [47]). The "momentum balance" equation has the form of Darcy's law relating the flow rate $U$ to the pressure $p$ :

$$
U=-\frac{k_{0} k(\sigma)}{\mu} \frac{\partial p}{\partial x}
$$

Here, $k_{0}$ is the absolute rock permeability and $k(\sigma)$ is the permeability reduction due to the retained particles $\sigma$. When expressed as a function of $\sigma$, it is called the formation damage function. It is normalized so that $k(0)=1$, i.e., it is one for clean porous rock. In general, the water viscosity $\mu$ can be considered constant for small suspended particle concentrations.

Equations (2.1)-(2.3) form a closed system of three equations for there unknowns $-c, \sigma$ and $p$. It is assumed that the filtration function is independent of the pressure $p$ : therefore, the first two equations decouple from the third and form a system for the two unknowns $c$ and $\sigma$. The physical domain is $t>0$ and $0<x<L$, where $L$ is the length of the core.

Remark 2.2. It is worth mentioning that the single capture mechanism expressed in system (2.1)-(2.2) may also be used to describe deep bed filtration with several simultaneous suspended particle capture mechanisms, such as size exclusion, attachment and gravity segregation ([17]). Some network models for deep bed filtration consider the situation where several particles must be deposited in a single pore until its complete plugging ([39]). In this case, all intermediate interactions "suspended particle - partly plugged pore" can also be aggregated into a single capture kinetics described by equation (2.2), see [37]. The system (2.1)-(2.2) can be obtained by averaging micro-scale stochastic equations for the size exclusion and the attachment mechanisms of particle retention ([38], [37]). For highly concentrated suspensions, the capture mechanism of bridging becomes dominant; the order of the "particle-pore reaction" may be much larger than one ([1]). In this case, the retention rate is not proportional to suspended concentration anymore. Modeling these processes is outside the of scope of the current paper.

2.1. Boundary and measured data. As initial data, we assume that the rock is clean and contains water with no particles; as boundary data, we assume that the solid particle concentration entering the porous medium is given, and that the effluent concentration is measured:

$$
\begin{array}{r}
\sigma(x, 0)=0 \quad \text { and } \quad c(x, 0)=0 \\
c(0, t)=c_{i}(t)>0, \quad t>0, \\
c(L, t)=c_{\exp }(t)>0, \quad t>0 .
\end{array}
$$

The pressure drop $\Delta p_{\text {exp }}=p(L, t)-p(0, t)$ is also measured in laboratory experiments. The quantity $\sigma(0, t)$, however, needs to be determined utilizing the model. Along the line $x=0$, we obtain from equations (2.2) and (2.5):

$$
\frac{d \sigma(0, t)}{d t}=\lambda(\sigma(0, t)) U c_{i}(t), \quad \text { and } \quad \sigma(0,0)=0,
$$

where $U$ is given. Integrating equation (2.7) provides $\sigma(0, t)$, which is positive and increasing. 
2.2. Solution for suspension flow. The well-posedness of the boundary/initial value problem (2.1)-(2.2) with boundary and initial data (2.4)-(2.5) was established in [3] and [4], where $U$ was taken as a constant and it was assumed that the filtration function $\lambda(\sigma)$ was $C^{1}$, i.e., it had one continuous derivative, and that $\lambda(\sigma)>0$ for $\sigma \in[0, \phi]$. A generalization of this result follows. For $t \leq(\phi / U) x, \sigma(x, t)$ and $c(x, t)$ vanish. For $t>(\phi / U) x$, one can rewrite equations $(2.1)-(2.2)$ on characteristic lines $x-(U / \phi) t=$ const in the form

$$
\begin{aligned}
& \frac{d \sigma}{d x}=-\lambda(\sigma) \sigma \\
& \frac{d c}{d x}=-\lambda(\sigma) c U .
\end{aligned}
$$

where $d / d x$ means differentiation along characteristic lines $x-(U / \phi) t=$ const ([3]). Since $c(0, t)=c_{i}(t)$ is specified and $\sigma(0, t)$ is obtained from solving equation (2.7), the family of equations (2.8)-(2.9) can be solved numerically using standard procedures for ODE's.

Although the non-linear inverse problem is inherently ill-posed, the following remark reflects that the direct problem is well-posed. This will be useful for proving that the regularized approximation of the inverse problem is well-posed, in the sense of Tikhonov, in some appropriate compact set (see Appendix A).

Remark 2.3. Since the solution $\sigma$ and $c$ of the system (2.8) and (2.9) are given by an ordinary differential equations along characteristic lines, the continuity of the solution is a consequence of the theorem on continuity of ODE solutions with respect to parameter changes (see [20], pag. 91). So the maps

$$
\lambda(\sigma ; \theta) \rightarrow \sigma(x, t ; \theta), \quad \lambda(\sigma ; \theta) \rightarrow c(x, t ; \theta) .
$$

are continuous in the uniform norm.

Differently from [3], where $\lambda(\sigma)$ was strictly positive, we assume that $\lambda(\sigma)$ is a nonnegative piecewise $C^{1}$ function that may vanish in its domain $0<\sigma<\phi$, i.e., $\lambda(\sigma)>0$ for $0 \leq \sigma<\sigma_{0}$ and $\lambda(\sigma)=0$ for $\sigma_{0} \leq \sigma<\phi$. The following Lemma, proved in [4], allows this assumption.

Lemma 2.4. The solution of (2.1)-(2.3) with data (2.4)-(2.5) is given by (2.8)-(2.9) in the trapezoidal domain $0 \leq x \leq L, 0<t \leq(\phi / U) x+\tau ; \tau$ can be infinite. If it is finite, $\sigma$ is constant and equal to $\sigma_{0}$ in the trapezoid $0 \leq(\phi / U) x+\tau \leq t$ and $c(x, t)=c_{i}(x-(U / \phi) t)$. Also, $c$ is continuous in the trapezoid $0 \leq x \leq L, t>(\phi / U) x$, and $\sigma$ is continuous in the infinite rectangle $0 \leq x \leq L, 0<t<\infty$.

In [3] and [4] the well-posedness of the direct problem was proved. To solve the inverse problems for the filtration function $\lambda(\sigma)$ and the formation damage function $k(\sigma)$, we need to solve equations (2.1)-(2.5) and (2.7) many times as part of an iterative optimization procedure. Thus, it is necessary to solve this system with high speed and accuracy.

\section{RECOVERY METHODS}

In this section, the values of the coefficients that parametrize the empirical functions are recovered by using optimization procedures to minimize functionals that represent the difference between the solution of the direct problem and the available experimental data. Taking into account that parameter estimation in systems of partial differential equations such as $(2.4)-(2.9)$ is usually unstable $([4],[13])$, i.e., the recovered parameters do not depend 
on the data in a stable way, we include a Tikhonov regularization term when necessary. This regularization enables one to obtain stable approximations of ill-posed inverse problems [43]. The well-posedness of the regularized approximation of the inverse problem of recovering the filtration and permeability reduction functions is proved in [4] in the operator theory framework; a summary is given here in Appendix A.

It is important to notice that the first two equations in the governing system, (2.1) and (2.2), are only weakly coupled to the last equation (2.3). Equations (2.1) and (2.2) determine the deposition of the suspended particles, while equation (2.3) relates the flow rate $U$ to the pressure drop through the loss of permeability, which depends on the deposited concentration. In fact, in laboratory experiments $U$ is measured, and the decoupling is complete. We are led naturally to split the inverse problem of determining the empirical functions into two separate problems.

First, we recover the filtration function using either the method presented below or the methods presented in [3] and [4]. This first inverse problem determines the filtration function from the outlet concentration, i.e., the kinetic particle capture rate is calculated from the particle concentration history. Then we recover the permeability reduction function using either the method presented below or the method presented in [4]. This second inverse problem determines the formation damage function from pressure drop, i.e., the dynamic coefficient that specifies the hydraulic conductivity increase due to particle retention is calculated from the history of the pressure loss on the core.

Remark 3.1. The inverse problem of determining the functions $\lambda(\sigma)$ and $k(\sigma)$ in deep bed filtration is analogous to that of determining the fractional flow function and the total mobility in two-phase transport in porous media. Fractional flow is determined from the fraction of water in the outlet two-phase flux $([46])$, while total mobility is determined from pressure drop and injection rate histories ([22]).

3.1. Optimization procedure. Good algorithms for constrained minimization are the essential tool for the development of efficient methods for general nonlinear programming ([15] and [30]). Such methods apply to problems of the form

$$
\text { find } x^{*}=\arg \min F(x) \text { subject to } x \in \Omega
$$

where $x^{*}, x \in \mathbb{R}^{n}$ and $n$ is the number of variables, $F(x): \mathbb{R}^{n} \rightarrow \mathbb{R}$ is a functional we wish to minimize, and $\Omega$ is a convex region in $\mathbb{R}^{n}$. In essence, these algorithms proceed as follows: at the $k$-th iteration, the current guess for the minimizer $x^{k} \in \Omega$ is incremented in the direction of $-\nabla F\left(x^{k}\right)$, yielding $\tilde{x}^{k}$; the size of the increment is determined from the difference $\left|F\left(x^{k-1}\right)-F\left(x^{k}\right)\right|$. Next, the resulting point is orthogonally projected onto the feasible set $\Omega$, yielding $\hat{x}^{k}$. The algorithm may search along the lines from $x^{k}$ to $\tilde{x}^{k}$ and $\hat{x}^{k}$ for better minimizers, i.e., linear searches may be conducted before or after the projection. This eventually brings $x^{k}$ to a local minimizer, either with $\nabla F=0$ or at the boundary $\partial \Omega$, where the algorithm stops: there is no guarantee the minimizer is unique or global.

This approach is particulary interesting if the projection onto $\Omega$ is easy to compute: it is trivial in the case where $\Omega \subset \mathbb{R}^{n}$ is defined by box boundaries $l_{i}<u_{i}, i=1, \ldots, n$. We used an implementation of the spectral projection gradient method with box constraints presented in detail in [8], developed by the very authors and very suitable to our problem. The code was designed for large problems (i.e., $n$ large) and is therefore extremely robust for a relatively simple application as the one presented in this work. The number $n$ of parameters to recover does not exceed three in the examples given in the following two sections. 
3.2. Recovering the filtration function. In this section we define our functional for solving the first inverse problem: namely, finding the filtration function from the effluent concentration history measured in laboratory experiments.

Before the recovery procedure, a parametrization $\lambda(\sigma ; \theta)$ must be chosen for the filtration function, where $\theta$ is the set of parameters. The form of these parametric functions and their parameter ranges are dictated by physical properties of the filtration function: concrete examples are discussed in the following two sections. Then we minimize a functional relating the filtration function and the effluent concentration:

$$
F^{c}(\theta, \alpha)=\int_{B}^{A}\left(c(L, t ; \theta)-c_{e x p}(t)\right)^{2} d t+\alpha^{2}\left\|\theta-\theta^{*}\right\|^{2} .
$$

In the first term of the right hand side, $c_{\text {exp }}(t)$ represents the effluent particle concentration history measured in the laboratory, $c(L, t ; \theta)$ is obtained by solving equations $(2.4)-(2.9)$ for a fixed set of parameters $\theta, B=(\phi / U) L$ is the breakthrough time and $A$ is the end time of the experiment. When necessary, we use the second term in the right hand side, which is a penalization term, where $\alpha$ is the Tikhonov regularization parameter. This term is required to obtain stable solutions only if the parametrization $\lambda(\sigma ; \theta)$ has an inadequate form or too many parameters; otherwise, the solution is regularized by the parametrization itself (see [4], page 146, or [16], [36]).

3.3. Recovering the permeability reduction function. For one-dimensional flow in a rock core, we divide equation $(2.3)$ by $1 / k(\sigma(x, t)) U$, integrate the resulting equation in $[0, L]$ and obtain the following relationship between deposited particle distribution and pressure drop history:

$$
-\int_{0}^{L} \frac{d x}{k(\sigma(x, t))}=\frac{k_{0}}{\mu U} \Delta p(t), \quad 0 \leq t \leq A .
$$

As in the previous subsection, first we choose a parametrization $k(\sigma ; \beta)$ for the permeability reduction function, with parameter set $\beta$. Then we minimize the functional:

$$
F^{p}(\beta, \gamma)=\int_{B}^{A}\left(\Delta p(t ; \beta)-\Delta p_{\exp }(t)\right)^{2} d t+\gamma^{2}\left\|\beta-\beta^{*}\right\|^{2}
$$

where $\Delta p(t ; \beta)$ is the right hand side of equation (3.2), $\Delta p_{\exp }(t)$ is the experimental data, and $\gamma$ is the regularization parameter. Once again, the penalization term is required to produce stable solutions in certain cases, e.g. when parameterization $k(\sigma ; \beta)$ is poorly chosen. Notice that the evaluation of $F^{p}(\beta, \gamma)$ requires the system of equations $(2.4)-(2.9)$ to be solved using the filtration function recovered from the effluent concentration history: once we have the deposition of particles $\sigma(x, t)$, then the integrals in equation (3.3) can be evaluated numerically.

Remark 3.2. The two inverse problems formulated in this section are consistent with the physical meaning of the unknown functions $\lambda(\sigma)$ and $k(\sigma)$. System $(2.1)-(2.2)$ describes the "kinematics" of deep bed filtration - retention kinetics between suspended and deposited concentrations. The unknown filtration function $\lambda(\sigma)$ is the probability for a particle to be captured during its travel along the unit length, i.e. $\lambda(\sigma)$ is a kinematic characteristic of the process, and it is determined from the outlet particle concentration $c(L, t)$.

The unknown permeability reduction function $k(\sigma)$ is a relative rate of particle suspension decrease under constant pressure gradient during the filtration. It is defined from the "momentum balance" equation (2.3) and it is therefore a dynamical property of deep bed 
filtration. It is determined from the histories of pressure drop on the core $\Delta p(t)$ and of flow rate $U(t)$, i.e., from dynamical data.

Granted this consistency, it is important to notice that no specific parametric form was imposed on either $\lambda$ or $k$ up to this point. The recovery procedure, although developed with this particular problem in view, is mathematically abstract and formally it makes no assumptions about the two functions to be recovered or about the input data. The optimization procedure, however, may impose restrictions: the procedure from [8] we chose requires that the functionals have continuous first-derivatives on $\theta$ and $\beta$, which is the case in the examples given in sections 4 and 5 .

\section{Numerical RESUlts With SYNThetic DATA}

In this section we present an example where the functionals (3.1) and (3.3) are minimized numerically. We use synthetic data $\widetilde{c_{e}}(t)$ and $\widetilde{\Delta p}(t)$ to calibrate the model and to test the algorithm. We define the parametric form of the filtration and permeability reduction functions as

$$
\lambda(\sigma)=\max \left\{0, \theta_{1}-\theta_{2} \sigma\right\} \quad \text { and } \quad k(\sigma)=\frac{1}{1+\beta \sigma},
$$

which are similar to those typically found in the literature ([31]). We fix $\theta_{1}=1, \theta_{2}=171$ and $\beta=300$ : these are the parameters we wish to recover after solving the inverse problem with the synthetic data. We also assume the inlet concentration $c_{i}(t)$ to be constant. The synthetic data are constructed in two steps. First, we solve the direct problem for some filtration and permeability damage functions; second, we add random perturbations to this exact solution to simulate observational error inherent to real data.

In order to avoid solving the inverse problem in a space with the same dimension and of the same type as the one in which the functions in (4.1) are defined (see [24] for a discussion on so-called inverse crimes), we fit rational functions $\tilde{\lambda}(\sigma)$ and $\tilde{k}(\sigma)$ to both $\lambda(\sigma)$ and $k(\sigma)$ in (4.1), obtaining

$$
\tilde{\lambda}(\sigma)=\frac{1-85.2 \sigma}{1+85.4 \sigma+15,737 \sigma^{2}} \quad \text { and } \quad \tilde{k}(\sigma)=\frac{1-149 \sigma}{1+151 \sigma-44,640 \sigma^{2}}
$$

that approximate $\lambda(\sigma)$ and $k(\sigma)$ given in (4.1), but have more parameters. We use the functions (4.2) to solve the direct problem given by the system of equations $(2.4)-(2.9)$ and the integral equation (3.2). Incidentally, the resulting profiles of $\widetilde{c_{e}}(t)$ and $\widetilde{\Delta p}(t)$ have maximum relative errors of $6.68 \times 10^{-3}$ and $4.72 \times 10^{-4}$, respectively, when compared to the solutions found using the prescribed functions (4.1).

We create four sets of perturbed synthetic data from the one single exact $\widetilde{c_{e}}(t)$ and $\widetilde{\Delta p}(t)$ solution obtained using $\tilde{\lambda}(\sigma)$ and $\tilde{k}(\sigma)$. More specifically, we introduce random perturbations of the order of $0.01,0.05,0.10$ and 0.15 , i.e., the relative error (noise/signal ratio) of the perturbed data is limited by these given values. The frequency of the added noise is similar to that observed in laboratory experiments $([1])$. These are shown in figure 4.1.

Solving the inverse problem for each of these four data sets, we recover the parameters for equations (4.1). Now we compare the unperturbed synthetic data to the results of the direct problem based on these recovered parameters, and find the relative error to be similar to the one between the unperturbed synthetic data and the solution obtained using the prescribed functions (4.1), as shown in table 4 . 


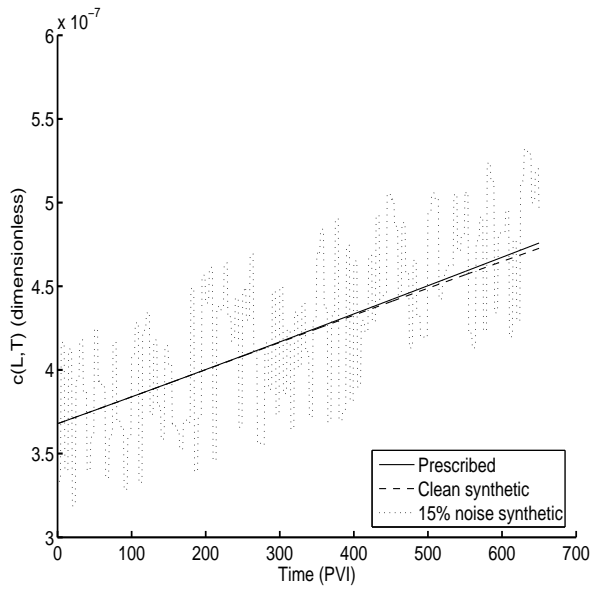

a) Effluent concentration

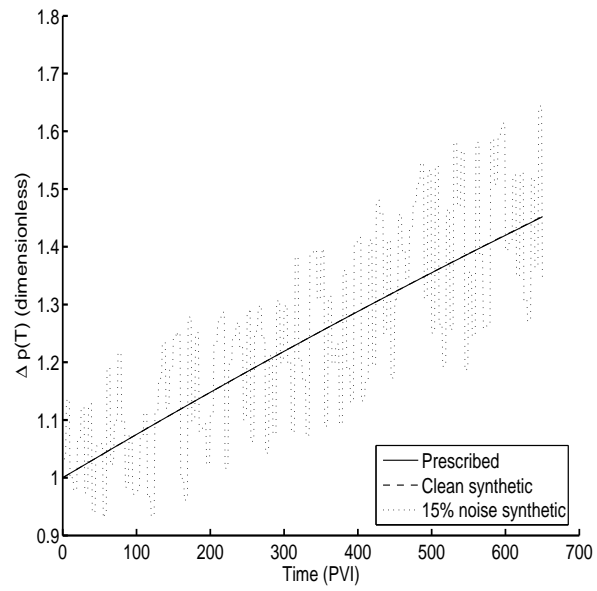

b) Pressure drop

Figure 4.1. Synthetic data.

\begin{tabular}{ccc} 
Noise & Max error in $c_{e}$ & Max error in $\Delta p$ \\
\hline $1 \%$ & $3.14 \times 10^{-3}$ & $5.21 \times 10^{-4}$ \\
$5 \%$ & $5.47 \times 10^{-3}$ & $1.32 \times 10^{-3}$ \\
$10 \%$ & $2.09 \times 10^{-2}$ & $1.58 \times 10^{-2}$ \\
$15 \%$ & $2.57 \times 10^{-2}$ & $1.70 \times 10^{-2}$
\end{tabular}

TABLE 4.1. Relative errors between the unperturbed synthetic data and the solutions obtained using the parameters recovered from the perturbed data sets.

In Figure 4.2 the corresponding recovered filtration and permeability reduction functions are shown. We see that these functions are recovered with reasonable accuracy, and smaller relative errors for cleaner data, as expected.

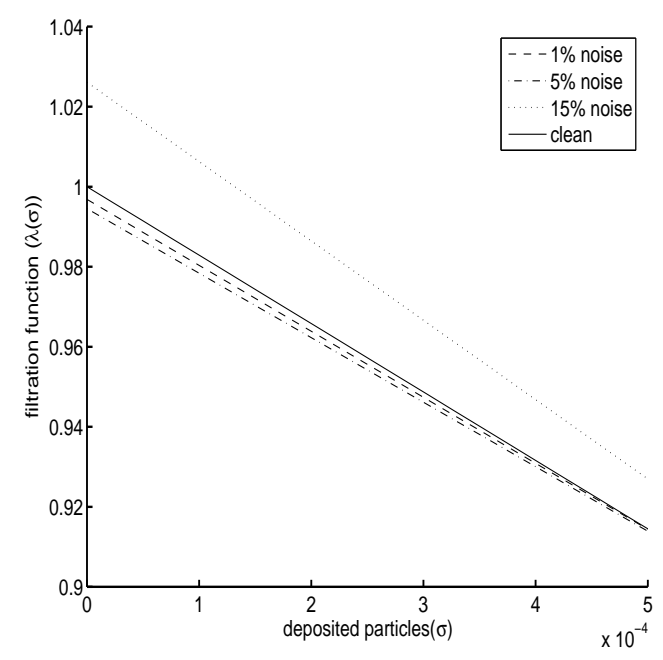

a) Filtration function

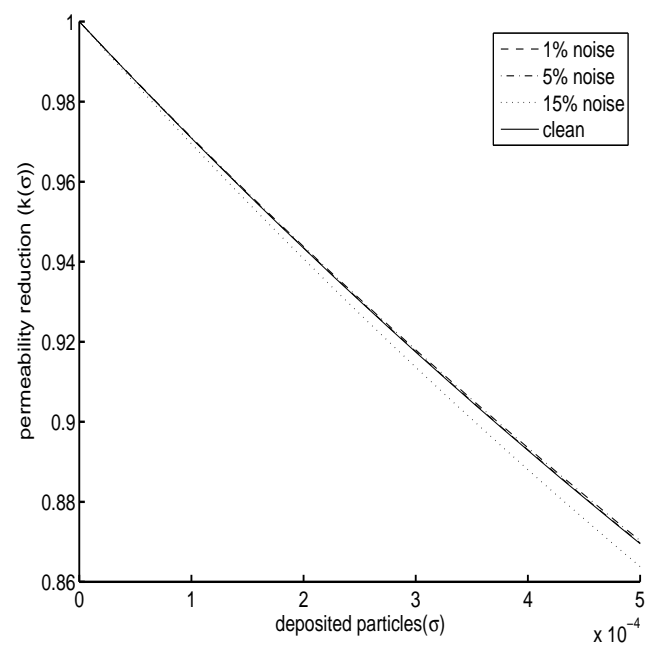

b) Permeability reduction function

FiguRE 4.2. Empirical functions recovered from noisy synthetic data. 
The numerical examples based on synthetic data suggest that the recovery method described here is appropriate for finding the permeability reduction and filtration functions from experimental data.

Remark 4.1. The optimizations were carried out using the code implemented by the authors of [8], after the algorithms detailed therein, which we introduced in Section 3. These algorithms implement many complementary minimization strategies and stopping criteria. Many of these criteria are based on values dependent on the scales of the problem, such as the magnitude of the functional being minimized or its gradient vector, and therefore we tuned the optimization constants to the scale of our data. The longest running time we experienced was a little over one minute on a $2 \mathrm{GHz}$ processor, with fifty evaluations of the cost functional. Since the complexity of the functional is related to the parametrization chosen, we obtain a similar performance when we apply the same procedures to experimental data, as detailed in the next section. This low computational cost is intrinsic to our recovery approach, and one of the advantages of the method.

4.1. Sensitivity analysis. Sensitivity analysis allows one to identify the "best" models, so that the number of parameters is neither insufficient nor is it so large as to render the solution of the parametrized inverse problem not unique. Also, once an optimal set of parameters is obtained, the computation of the corresponding sensitivity matrix allows studying the stability of the proposed inverse method ([41]). Here we perform the sensitivity analysis only for the filtration function given in (4.1).

To calculate the sensitivity matrix, we use the analytical solution of system (2.1)-(2.2). For simplicity, we transform the physical domain into dimensionless coordinates by the scaling equations

$$
X=\frac{x}{L} \quad \text { and } \quad T=\frac{U}{\phi L} t
$$

so $X$ is in the $[0,1]$ range; one unit of dimensionless time $T$ is how long it takes to inject a volume of fluid corresponding to the porous volume of the sample, hence the term porous volume injected (PVI). We also define $\sigma(X, T) \equiv \phi^{-1} \sigma(x, t)$ and $\lambda(\sigma(X, T)) \equiv \phi L \lambda(\sigma(x, t))$, so that the equations (2.1)-(2.2) become

$$
\begin{gathered}
\frac{\partial}{\partial T}(c+\sigma)+\frac{\partial c}{\partial X}=0 \\
\frac{\partial \sigma}{\partial T}=\lambda(\sigma) c .
\end{gathered}
$$

The solution for the system (4.4)-(4.5) with $\lambda(\sigma)$ given by equation (4.1), derived in [4], is

$$
\begin{array}{rlr}
\sigma(X, T ; \theta)=0, c(X, T ; \theta)=0 & \text { for } \quad T<X, \\
\sigma(X, T ; \theta)=\frac{\theta_{1}}{\theta_{2}}\left[1+\frac{e^{-c_{i 0} \theta_{2} T} e^{\left(\theta_{1}+c_{i 0} \theta_{2}\right) X}}{1-e^{-c_{i 0} \theta_{2}(T-X)}}\right]^{-1} & \text { and } & \\
c(X, T ; \theta)=\frac{c_{i 0} \theta_{2} \sigma(X, T)}{\theta_{1}\left(1-e^{-c_{i 0} \theta_{2}(T-X)}\right)} \quad \text { for } & T>X .
\end{array}
$$


We wish to study the sensitivity of the recovered parametric function $c(1, T ; \theta)$; the coefficients of the sensitivity matrix (see [41] and [42]) are defined by

$$
S_{\theta_{i}}(T)=\frac{\partial c(1, T ; \theta)}{\partial \theta_{i}}
$$

For a fixed $\theta$, the magnitude of the function $S_{\theta_{i}}(T)$ reflects the sensitivity of the solution with respect to the $i$-th parameter at a particular time. The sensitivity coefficients for the filtration function given in equation (4.1) are

$$
S_{\theta_{1}}=\frac{-c_{i o} e^{-c_{i o} \theta_{2}(T-1)} e^{\theta_{1}}}{\left(1+e^{-c_{i o} \theta_{2}(T-1)}\left(e^{\theta_{1}}-1\right)\right)^{2}}, \quad S_{\theta_{2}}=\frac{c_{i o}^{2}(T-1) e^{-c_{i o} \theta_{2}(T-1)}\left(e^{\theta_{1}}-1\right)}{\left(1+e^{-c_{i o} \theta_{2}(T-1)}\left(e^{\theta_{1}}-1\right)\right)^{2}} .
$$

From (4.10) we obtain

$$
S_{\theta_{1}} / S_{\theta_{2}}=-\frac{e^{\theta_{1}}}{c_{i o}\left(e^{\theta_{1}}-1\right)(T-1)} .
$$

Equation (4.11) implies that the sensitivity of $c(1, T ; \theta)$ is different relatively to $\theta_{1}$ and $\theta_{2}$; as a function of $T>1$, the solution is relatively more sensitive to $\theta_{1}$ for smaller values of $T$. In the particular example we developed in this section, the values of $S_{\theta_{i}}$ are such that $\left|c(1, T ; \theta) / S_{\theta_{1}}(T)\right| \approx 1$ and $\left|c(1, T ; \theta) / S_{\theta_{2}}(T)\right| \approx 10^{3}$ in the time interval $1<T<650$, i.e., perturbations of $\theta_{1}$ will reflect on $c(1, T ; \theta)$ with the same order of magnitude, whereas the effects of perturbations of $\theta_{2}$ are reduced by a factor of $10^{-3}$. Put simply, this solution is sensitive to the first parameter $\theta_{1}$, and not to $\theta_{2}$. These facts discourage the use of more parameters for the filtration function. A two-parameter, non-constant $\lambda(\sigma)$ such as the ones used in this section and in the next, is enough to model most of the experimental data we have analysed. In the last examples in Section 5, we use three-parameter filtration functions to account for a different behaviour of the effluent concentration history.

A similar analysis can be conducted for the pressure drop history $\Delta p(T ; \theta, \beta)$. However, $\Delta p$ is a function of $\sigma(X, T ; \theta)$ (see equation (3.2)); therefore, the observations regarding the sensitivity of $c(1, T ; \theta)$ are applicable to $\Delta p$ as well. For the experiment presented in this section, the numerical value of the sensitivity coefficient $\partial(\Delta p) / \partial \beta$ is two orders of magnitude smaller than the actual value of $\Delta p$, so the pressure drop is rather insensitive to this single parameter.

\section{NumERICAL RESUltS FOR EXPERIMENTAL DATA}

Data from Kuhnen et alii. We applied the method to the effluent particle concentration experimental data described by Kuhnen et alii in [26], shown in Figure 5.1. In these experiments, hematite suspensions of equal concentration and varying ionic strengths were injected at an equal rate into sandstone, to investigate the relation between filtration phenomena and electrostatic attraction between oppositely charged particle and porous medium surfaces. No cake formation was observed in these experiments.

In order to apply our empirical model, we tested numerous parametrizations on each of the six data series contained therein, and obtained the following results.

All the data series show an increasing trend. The first three of them go close to the zero filtration rate situation, where $c_{e}=c_{i}$ and $\lambda(\sigma)=0$. For the model to reproduce the first case accurately, i.e., to approach $\lambda(\sigma)=0$ as $\sigma$ increases, this trend must be taken into account: all successful parametrizations were of the form $\lambda_{0} e^{-f(\sigma)}$, where $f(\sigma)$ was a nonnegative increasing function. Polynomials of various degrees yielded different results: the 


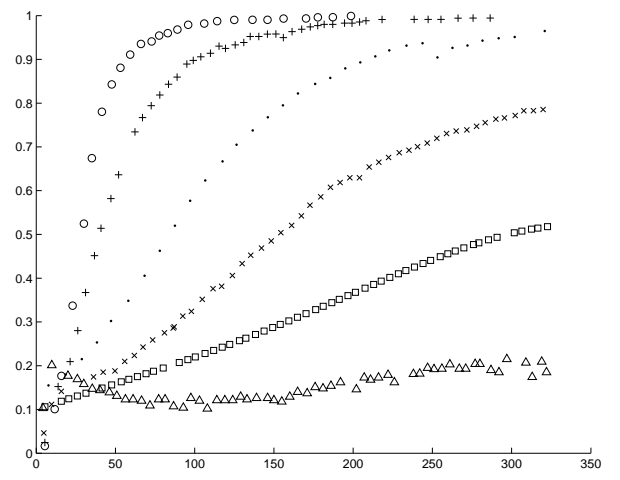

FiguRE 5.1. Effluent concentration histories from [26], up to 300 PVI. We will refer to these six series by number, from top to bottom.

best all-around parameterization was $\lambda_{0} e^{-\theta \sigma^{2}}$, for all six data series. For the latter times of the first two series, in the late time ranges where $c_{e} \approx c_{i}$, a good match was only obtained using $f(\sigma)=\theta \sigma^{4}$.

We remark that the method proposed in [3] does not work for the zero filtration rate situation observed in the first three data sets, as the quantity actually recovered is $1 / \lambda(\sigma)$ rather than $\lambda(\sigma)$. The remaining three do not approach this limit.

Figure 5.2 shows good matches obtained using the quadratic exponent. Figure 5.3 shows the difference of the two best parameterizations for series approaching the limit $c_{e} / c_{i} \cong 1$.

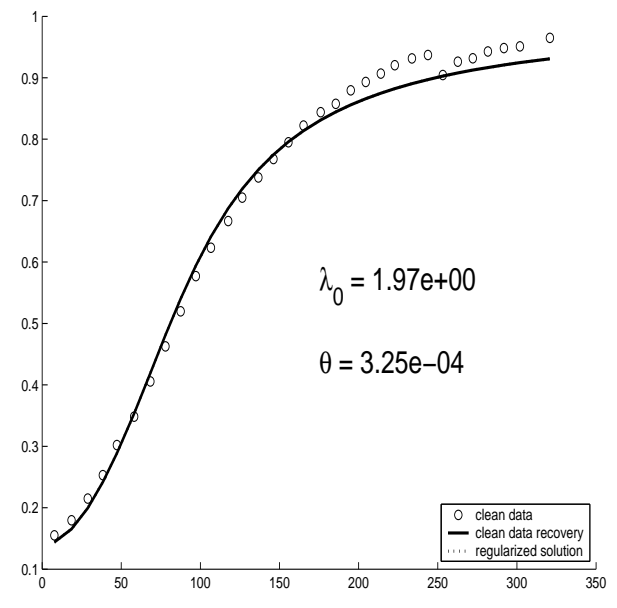

a) Data series 3

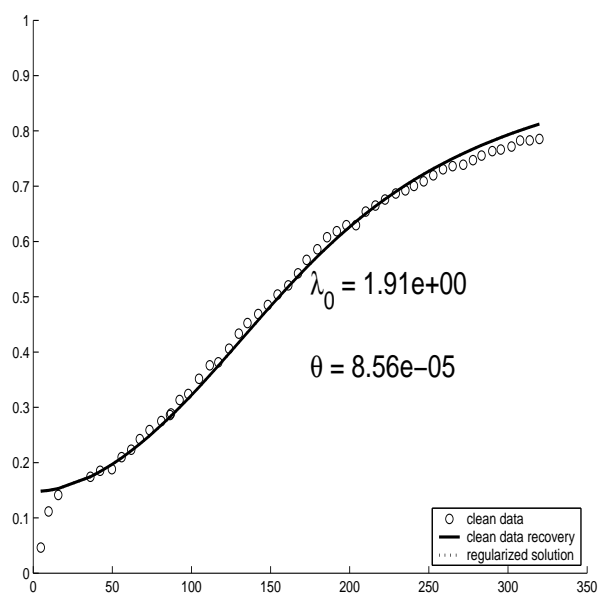

b) Data series 4

FiguRE 5.2. Best fits for effluent concentration using $\lambda(\sigma)=\lambda_{0} e^{-\theta \sigma^{2}}$.

Restricting the input data to the first 70 PVI of the last four data series, shown as markers in Figure 5.4a, we recovered the filtration functions shown in Figure 5.4b. We calculated the effluent concentrations using these filtrations and plotted them over the input data in Figure 5.4a, with which they coincide visually.

Data from Soma and Papadopoulos. Soma et al. performed a series of four experiments injecting oil-in-water emulsions into quartz sand (see [40]), using similar conditions 


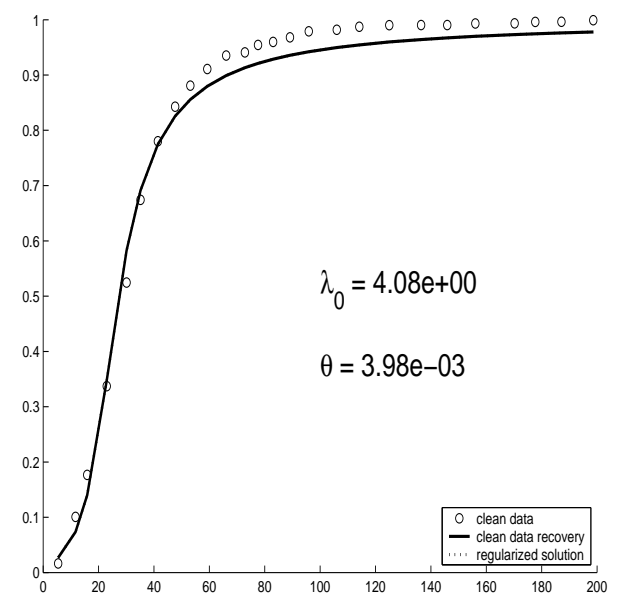

a) $\lambda(\sigma)=\lambda_{0} e^{-\theta \sigma^{2}}$

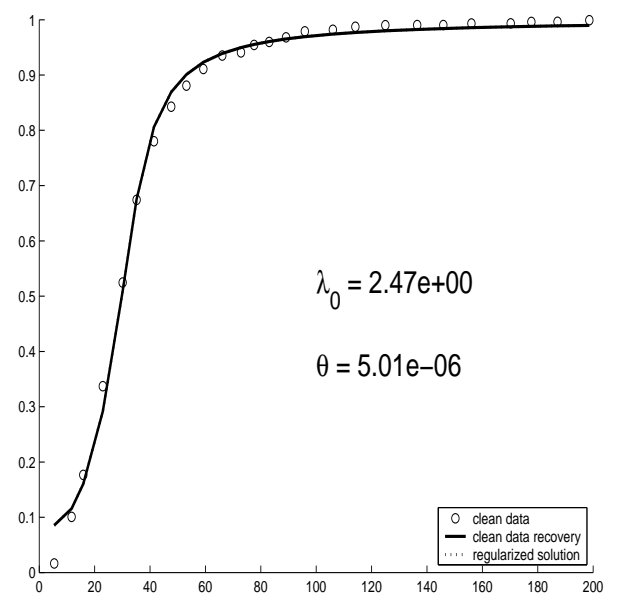

b) $\lambda(\sigma)=\lambda_{0} e^{-\theta \sigma^{4}}$

Figure 5.3. Profiles recovered using two fits for $\lambda$ in data series 1 .

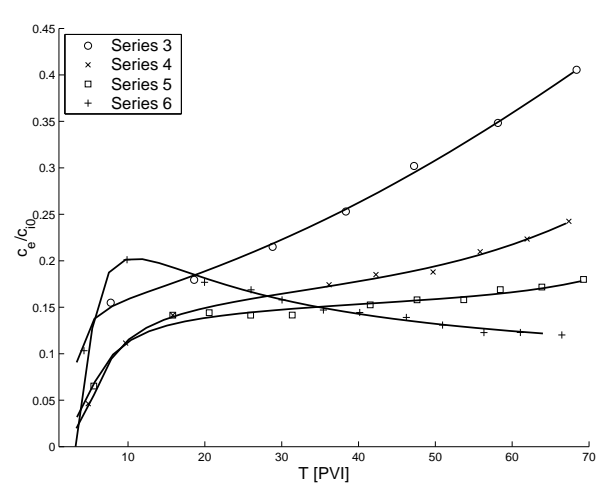

a) Cropped data and model prediction.

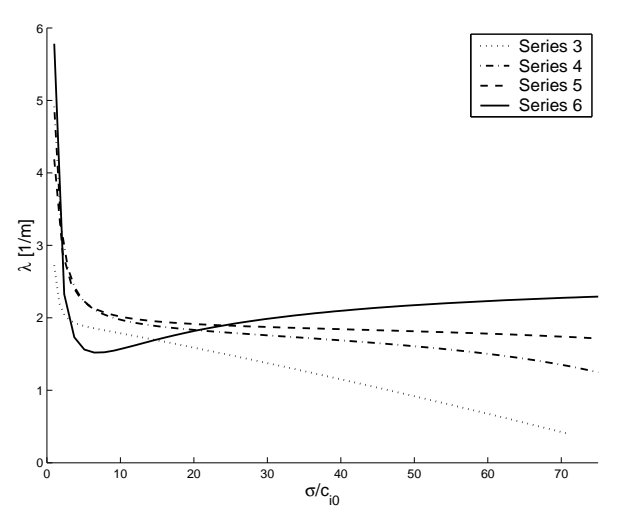

b) Corresponding filtration functions.

FiguRE 5.4. Excellent recovery obtained using the optimization solution shown over smooth approximations of the actual data.

and varying the ionic strength of the emulsion, for which they measured both effluent concentration and total permeability reduction, i.e., the pressure drop history. There was no cake formation in their experiments.

We applied the empirical model (2.1)-(2.3) to the two experiments with higher ionic strength, where there was enough deposition both to prevent the effluent concentration curve from reaching $c_{e} / c_{i} \approx 1$ quickly, and to produce significant permeability reduction.

However, the effluent concentration history curves are not monotone in these two experiments: this is another situation where the method presented in [3] fails to produce good results. The authors [40] attribute this lack of monotonicity to a greater attraction of oil droplets in the suspension to previously deposited oil rather than to the bare pore surface. In terms of the empirical $\lambda(\sigma)$ coefficient, this translates into a non-monotone behaviour of the filtration function, one that increases after a certain value of $\sigma$ is reached. To account for this behavior, we chose the parametrization

$$
\lambda(\sigma)=\theta_{1}\left(e^{-\theta_{2} \sigma^{2}}+\theta_{3} \sigma\right) ; \quad \theta_{1}, \theta_{2}, \theta_{3}>0 .
$$


For the permeability reduction function, we use the inverse of a second degree polynomial, which is compatible with the literature ([31]) and suitable for our calculations. Higher degree polynomials did not yield better results.

For both data sets, the model reproduced well both the effluent concentration and permeability reduction curves, as shown in Figure 5.5. The parametric empirical functions recovered solving the inverse problems are shown in Figure 5.6.

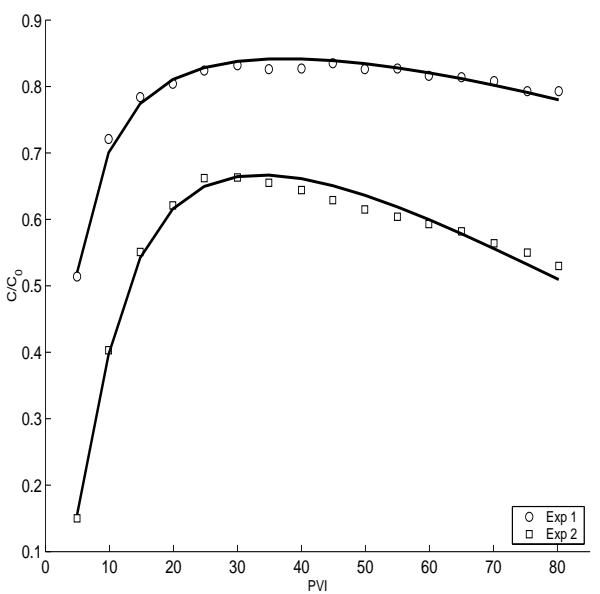

a) Effluent concentration

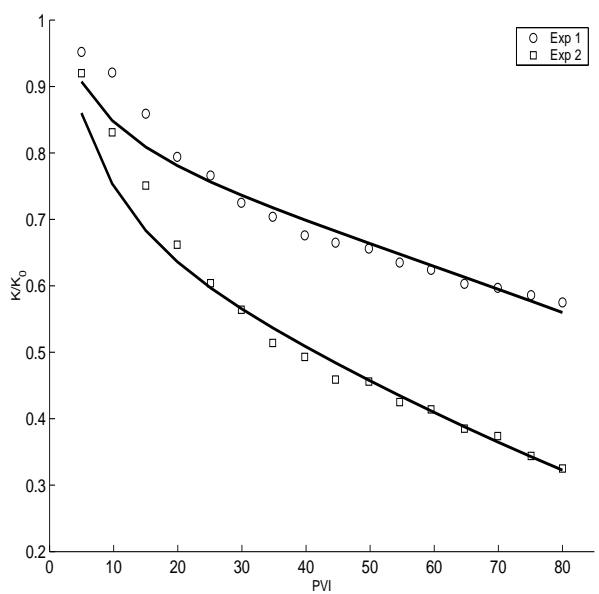

b) Permeability reduction

FiguRE 5.5. Recovered histories and original data in two experiments.

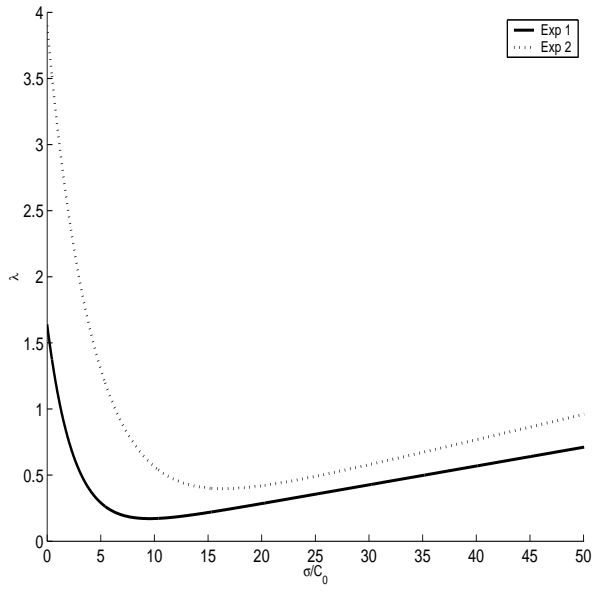

a) Filtration function

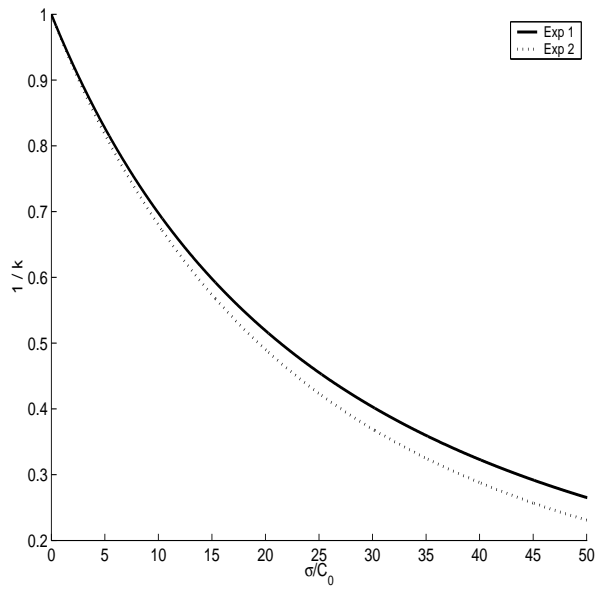

b) Permeability reduction function

FiguRE 5.6. Recovered empirical functions in two experiments.

\section{Conclusion}

The recovery method described here consists of solving two inverse problems: in the first one the filtration function is determined from the effluent concentration evolution, and in the second one the formation damage function is determined from the pressure drop history. In the cases examined, the method yields good matches between synthetic/experimental and predicted data. 
The results given in Sections 4 and 5 indicate that the method is robust and flexible, for it allows the analysis of experimental data that the other recovery methods in the literature cannot analyze. The method proposed in [3] requires that $\lambda(\sigma)$ does not approach zero, i.e., it cannot deal with data where $c_{e} / c_{i}$ approaches one. The three-point method presented in [5] and [6] assumes $\lambda(\sigma)$ to be a constant $\lambda_{0}$, which is equivalent to assuming that $c_{e} / c_{i}$ is constant in time after breakthrough: this is not the behaviour observed in any of the experiments presented in Section 5. The distinguishing feature of the method presented in this work is that it imposes few restrictions on the form of the functions to be recovered, providing a flexible tool for studying experimental data. At the same time, it is easier to implement than other methods equally adequate for practical purposes such as the method for recovering the permeability reduction developed in [4]. It is also flexible in the sense that, by accommodating different physical conditions under a minimal set of macroscopic parameters, all built into the recovered permeability damage and filtration functions, it can be readily applied to variations of the model (2.1)-(2.3) which cannot be solved numerically by $(2.8)-(2.9)$.

One must keep in mind, however, that the method assumes that both functions to be recovered can be represented by simple parametric expressions, and that the phenomena conforms to all modeling assumptions made in [19] and the other works derived from there, based on which this inverse problem was formulated and solved. As this happens to be the case in many real industrial applications, the method proposed here is viable for determining the filtration and formation damage functions from laboratory corefloods for use in predicting the injectivity decline, formation damage and contaminant propagation in a variety of petroleum and environmental engineering projects.

We did not fully automate the method: at this stage, part of the recovery procedure is a skillful choice of parametrizations, which requires good understanding of the physics related to the experimental data. Full automation would require analyzing dozens of varied cases. This is not warranted yet, as the model (2.1)-(2.3) may not be valid for all filtration problems.

\section{ApPendix A. WELL-POSEDNESS OF THE REGULARIZED INVERSE FILTRATION PROBLEM}

Mathematical problems that are supposed to be solved using real data containing errors must be well-posed, i.e., $\mathcal{O}(1)$ perturbations cannot arise in the answer from infinitesimal perturbations of the data. In this section we prove that the inverse problem formulated in equation (3.1) is well-posed in the sense of Tikhonov (see [27]). To do so, a feasible subset of parameters is chosen, such that the minimization problem has a unique minimum, and that small perturbations of experimental data produce small parameter variations. We rewrite the inverse problem in the framework of operator theory. Several results on regularization of non-linear operators are used to prove the well-posedness of the inverse problem (see [14]).

The stability and convergence results obtained here are based on [7], [14], [25] and [9]. We choose

$$
D\left(G^{c}\right)=\left\{\lambda \in H^{2}[0,1], \quad \text { such that } \lambda>\bar{\lambda}_{1}\right\},
$$

with $\bar{\lambda}_{1}$ constant. Here, $H^{2}[0,1]$ is the Hilbert space of functions defined in the interval $[0,1]$ with norm

$$
\|f\|^{2}=\int_{0}^{1}\left(|f(x)|^{2}+\left|f^{\prime}(x)\right|^{2}+\left|f^{\prime \prime}(x)\right|^{2}\right) d x .
$$


Let us define the non-linear operator

$$
G^{c}: D\left(G^{c}\right) \subset H^{2}[0,1] \rightarrow L^{2}[0, A], \quad G^{c}(\lambda)=c(1, \cdot ; \lambda),
$$

where $\lambda$ represents the filtration function and $c(1, \cdot ; \lambda)$ is the effluent concentration obtained from the solution of the system $(2.5),(2.8)-(2.9)$. Now $L^{2}[0,1]$ is the Hilbert space of functions defined in the interval $[0,1]$ with norm

$$
\|f\|^{2}=\int_{0}^{1}|f(x)|^{2} d x
$$

From the well-posedness of the direct problem ([4]), for each $\lambda$ there exists a unique function $c(1, \cdot ; \lambda)$, so the operator in (A.2) is well-defined. Notice that the domain $D\left(G^{c}\right)$ is closed and convex, therefore it is weakly closed. Let us define

$$
\mathcal{M}=\left\{\lambda \in H^{2}[0,1] \quad \text { such that } \quad \bar{\lambda}_{2}<\lambda<\bar{\lambda}_{3}\right\}
$$

where $\bar{\lambda}_{2}$ and $\bar{\lambda}_{3}$ are constants.

We recall the definition of $H^{s}[0,1]$ when $s$ is not integer: Let $a_{n}$ be the Fourier coefficient of a function in $[0,1]$, namely

$$
a_{n}=\int_{0}^{1} e^{-2 \pi n x i} f(x) d x
$$

The norm in $H^{s}[0,1]$ is

$$
\|f\|=\left(\sum_{n=-\infty}^{\infty}\left(1+|n|^{2 s}\right)\left|a_{n}\right|^{2}\right)^{1 / 2} .
$$

We have following:

Theorem A.1. Let $\lambda$ represent the filtration function and $c(1, \cdot ; \lambda)$ the solution of the system (2.1)-(2.2), with initial and boundary condition given in (2.4) and (2.5). Let $\eta \geq 0$ and consider the domain

$$
D_{\eta}\left(G^{c}\right)=\left\{\lambda \in H^{2+\eta}[0,1] \text { such that } \lambda>\bar{\lambda}_{2}\right\} .
$$

The following assertions are valid.

i) The (non-linear) operator

$$
G^{c}: D\left(G^{c}\right) \subset H^{2+\eta}[0,1] \rightarrow L^{2}[0, A], \quad G^{c}(\lambda)=c(1, \cdot ; \lambda),
$$

is continuous and injective.

ii) Let $D\left(G^{c}\right)$ be as in equation (A.1). Then the operator in (A.2) is weakly closed and compact.

iii) The map $G^{c}: \mathcal{M} \rightarrow G^{c}(\mathcal{M})$ is continuous and has continuous inverse.

Proof: (i) Let $\lambda_{n} \rightarrow \lambda$ in $H^{2+\eta}[0,1]$ with $\eta \geq 0$. Since $H^{2+\eta}$ is compactly embedded in $C^{1}[0,1]$ then $\lambda_{n} \rightarrow \lambda$ uniformly in $C^{1}[0,1]$, from Remark 2.3 it follows that $G^{c}\left(\lambda_{n}\right) \rightarrow G^{c}(\lambda)$ in $L^{2}[0, A]$. The injectivity is a consequence of the uniqueness of the solution of the system of equations (2.1)-(2.5) and (2.7).

(ii) Let $\left\{\lambda_{n}\right\}$ be a sequence in $D\left(G^{c}\right)$ converging weakly in $H^{2}[0,1]$ towards $\lambda$. Since 
$D\left(G^{c}\right)$ is weakly closed, then $\lambda \in D\left(G^{c}\right)$ and since $H^{2}[0,1]$ is compactly embedded in $C^{1}[0,1]$, which is the Banach space of functions with continuous derivatives and norm $\|f\|=\max _{[0,1]}|f(x)|+\max _{[0,1]}\left|f^{\prime}(x)\right|$, then $\lambda_{n} \rightarrow \lambda$ in $C^{1}[0,1]$. By (i) $G^{c}\left(\lambda_{n}\right) \rightarrow G^{c}(\lambda)$ in $L^{2}[0, A]$. Thus, $G^{c}$ is compact, hence weakly closed.

(iii) Notice that $G^{c}$ is continuous in $\mathcal{M}$ by (ii). Moreover, $\mathcal{M}$ is a compact subset of $C[0,1]$, because it consists of uniformly bounded functions in $H^{1}[0,1]$ ([11]). Therefore (iii) is a consequence of the Lemma of Tikhonov.

From (ii) in Theorem $A .1$ and Proposition 10.1 in [14], the inverse problem of determining the filtration function $\lambda$ in $G^{c}(\lambda)=b$ with given $b=c_{e}(\cdot)$ is locally an ill-posed problem. Hence, the Regularization of Tikhonov is used to find stable solutions. The regularized solution is determined as the minimizer over $D\left(G^{c}\right)$ of the functional

$$
\lambda \rightarrow\left\|G^{c}(\lambda)-c_{e}(\cdot)\right\|_{L^{2}[0, A]}^{2}+\alpha^{2}\left\|\lambda-\lambda^{*}\right\|_{H^{1}[0,1]}^{2},
$$

where $\alpha$ is the regularization parameter. Since $G^{c}$ is weakly closed, stability and convergence of the regularized solution follow from Theorem 10.2 and 10.3 in [14].

Let us denote by $\theta$ a parametrization of the filtration function $\lambda(\sigma)$. Neglecting the interaction between the parameters, i.e., by assuming that they are uncorrelated, we obtain

$$
\left\|\lambda-\lambda^{*}\right\|_{H^{1}[0,1]}^{2} \approx\left\|\theta-\theta^{*}\right\|^{2}
$$

where $\|\cdot\|$ denotes some appropriate norm in the parameter space. Thus, from equation (A.7), we see that the penalization functional can be written in terms of the parameters $\theta$.

Acknowledgments. The authors are grateful to Alexandre G. de Siqueira, Antonio Luiz Serra and Cláudio Furtado for encouragement and support during the solution of this problem and for many useful discussions. This work was supported in part by CNPq under Grants 301532/2003-06 and 141298/2001-04; FAPERJ under Grants E-26/150.408/2004 and E-26/150.163/2002.

\section{REFERENCES}

[1] F. A. H. Al-Abduwani. Investigation into the Injectivity Decline Due to Produced Water Re-Injection under Fracturing Conditions. PhD thesis, TU Delft, Netherlands, 2005.

[2] J. E. Altoe, P. G. Bedrikovetsky, A. L. Siqueira, and F. Shecaira. Correction of basic equations for deep bed filtration with dispersion. Journal of Petroleum Science and Engineering, 51:68-84, 2006.

[3] A. C. Alvarez, P. G. Bedrikovetsky, G. Hime, A. O. Marchesin, D. Marchesin, and J. R. Rodriguez. A fast inverse solver for the filtration function for flow of water with particles in porous media. Inverse Problems, 22:69-88, 2006.

[4] A.C. Alvarez. Inverse problems for deep bed filtration in porous media. PhD thesis, IMPA. Brasil, 2005. Available online at http://www.impa.br.

[5] P.G. Bedrikovetsky, D. Marchesin, F. Shecaira, A. L. Serra, and E. Resende. Characterization of deep bed filtration system from laboratory pressure drop measurements. Journal of Petroleum Science and Engineering, 64(3):167-177, 2001.

[6] P.G. Bedrikovetsky, K. Tran, W.M.G.T. Van den Broek, D. Marchesin, E. Rezende, A. Siqueira, A.L. Serra, and F. Shecaira. Damage characterization of deep bed filtration from pressure measurements. Journal SPE PF, 3:119-128, 2003.

[7] A. Binder, W. H. Heinz, C. W. Groetsch, A. Neubauer, and O. Scherzer. Weakly closed nonlinear operators ans parameter identification in parabolic equations by tikhonov regularization. Applicable Analysis, 55:215-234, 1994. 
[8] E. G. Birgin, J. M. Martínez, and M. Raydan. Nonmonotone spectral projected gradient methods on convex sets. SIAM. Journal on Optimization, 10:1196-1211, 2000.

[9] A. Colonius and Kunisch K. Output least square stability in elliptic system. Appl. Math. Optim., 19:3363, 1989.

[10] M. Y. Corapcioglu and H. Choi. Modeling colloid transport in unsaturated porous media and validation with laboratory column data. Water Resource Research, 32(12):3437-3449, 1996.

[11] J. Cullum. Numerical differentiation and regularization. SIAM, J. Numer. Anal, 8(2):337-344, 1971.

[12] G. Elimelech, J. Gregory, X. Jia, and R. A. Williams. Particle Deposition and Aggregation. ButterworthHeinemann, USA, 1995.

[13] H. W. Engl. Regularization methods for the stable solution on inverse problems. Surv. Math. Ind., 3:7-143, 1993.

[14] H. W. Engl, M. Hanke, and A. Neubauer. Regularization of Inverse Problems. Kluwer Academic Publishers, 2000.

[15] A. Friedlander and J. M. Martinez. On the maximization of a concave quadratic function with box constraints. SIAM. Journal on Optimization, 4:177-192, 1994.

[16] C. W. Groetsch. The Theory of Tikhonov regularization for Fredholm Equations of the First Kind. Pitman, Boston, 1984.

[17] R. G. Guedes, F. A. H. Al-Abduwani, P. G. Bedrikovetsky, and P. K. Currie. Injectivity decline under multiple particle capture mechanisms. In SPE International Symposium and Exhibition on Formation Damage Control, February 2006.

[18] Al-Abduwani F. A. H., R. Farajzadeh, and Broek W. M. G. T. van den and Currie P. K. Utilizing static filtration experiments to test existing filtration theories for conformance. In Second Produced Water Workshop. Aberdeen, UK, 2004.

[19] J. P. Herzig, D.M. Leclerc, and P. Le. Goff. Flow of suspensions through porous media-application to deep filtration. Industrial and Engineering Chemistry, 65(5):8-35, 1970.

[20] E. Hille. Lectures Notes on Ordinary Differential Equation. Addison-Wesley Pub. Co, 1969.

[21] T. Iwasaki. Some notes on sand filtration. Jour. Am. Water Works Assoc., 29(10):1591-1602, 1937.

[22] E.F. Johnson, D.C. Bossler, and V.O. Naumann. Calculation of relative permeability from displacement experiments. J. Petr. Tech., 11:61-63, 1959.

[23] P. R. Johnson and M. Elimelech. Dynamics of colloid deposition in porous media: Blocking based on random sequential adsorption. Langmuir, 11:801-812, March 1995.

[24] J. P. Kaipio and E. Somersalo. Statistical inverse problems: Discretization, model reduction and inverse crimes. to appear in J. of Comp. Appl. Math, 2006.

[25] C. Kravaris and J. H. Seinfeld. Identification of parameters in distributed parameter system by regularization. SIAM J. control and Optimization., 23(2), 1985.

[26] F. Kuhnen, K. Barmettler, S. Bhattacharjee, M. Elimelech, and R. Kretzschmar. Transport of iron oxide colloids in packed quartz sand media: Monolayer and multilayer deposition. Journal of Colloid and Interface Science, 231:32-41, 2000.

[27] M.M. Lavrent'ev and L. Ya. Savel'ev. Linear Operators and ill-posed problems. Spring Sheet, NY, 1991.

[28] B. E. Logan, D. G. Jewett, R. G. Arnold, E. J. Bouwer, and O'Melia C. R. Clarification of clean-bed filtration models. Journal of Environmental Engineering, 121:869-873, 1995.

[29] J. D. Logan. Transport Modelinig in Hydrogeochemical Systems. Springer, 2001.

[30] J. M. Martinez. Augmented lagrangians and sphere packing problems. International Journal of Computer Mathematics, 70:75-86, 1998.

[31] L. M. McDowell-Boyer, J. R. Hunt, and N. Sitar. Particle transport through porous media. Water Resources Research, 22(13):1901-1921, 1986.

[32] S. Pang and M. M. Sharma. A model for predicting injectivity decline in water injection wells. In 69 th Annual Technical Conference and Exhibition. New Orleans. Society of Petroleum Engineers. SPE 28489, 1994.

[33] A. S. Payatakes, R. Rajagopalan, and C. Tien. Application of porous medium models to the study of deep bed fitration. The Canadian Journal of Chemical Engineering, 52, December 1974.

[34] R. Rajagopalan and C. Tien. Trajectory analysis of deep-bed filtration with the sphere-in-a-cell porous media model. AIChe Journal, 22:523-533, 1976. 
[35] C. Roque, G. A. Chauveteau, G. Thibault, and M. Bouteca. Mechanisms of formation damage by retention of particles suspended in injection water. In SPE paper 30110, 1995.

[36] E. Schock. On the asymptotic order of accuracy of tikhonov regularizations. Journal of Optimization Theory Appl., 44:95-104, 1984.

[37] A. A. Shapiro, P. G. Bedrikovetsky, A. Santos, and O. O. Medvedev. A stochastic model for filtration of particulate suspensions with incomplete pore plugging. Journal of Transport in Porous Media, 2006.

[38] M. M. Sharma and Y. C. Yortsos. Transport of particulate suspensions in porous media: Model formulation. AIChe Journal, 33(10):1636-1643, 1987.

[39] A. G. Siqueira, E. Bonet, and F. S. Shecaira. Network modeling for transport of water with particles in porous media. In SPE Latin America and Caribbean Petroleum Engineering Conference, April 2003.

[40] J. Soma and K.D. Papadopoulos. Flow of dilute, sub-micron emulsions in granular porous media: effects of ph and ionic strength. J. Colloids and Surfaces. Series A: Physicochemical and Engineering Aspects, 101:51-61, 1995.

[41] N. Sun, N. Z. Sun, M. Elimelech, and N. Ryan. Sensitivity analysis and parameter identifiability for colloid transport in geochemically hererogeneous porous media. Water Resources Research, 37(2):209$222,2001$.

[42] A. Tarantola and B. Valette. Generalized nonlinear inverse problem solved using the least squares criterion. Rev. Geophys. Space Phys., 20:219-232, 1982.

[43] A. N. Tikhonov and V. Y. Arsenin. Solutions of Ill-Posed Problems. Wiley, New York, 1977.

[44] N. Tufenkji, J. A. Redman, and M. Elimelech. Interpreting deposition patterns of microbial particles in laboratory-scale column experiments. Environmental Sciences Technology, 37:616-623, 2003.

[45] J. P. Veerapen, B. Nicot, and G. A. Chauveteau. In-depth permeability damage by particle deposition at high flow rates. In SPE paper 68962, 2001.

[46] H.J. Welge. A simplified method for computing oil recovery by gas or water drive. Trans. AIME, 195:9198, 1952.

[47] K. I. Wennberg and M. M. Sharma. Determination of the filtration coefficient and the transition time for water injection wells. In Society of Petroleum Engineers. SPE 38181., 1997. 